\title{
The pathway toward the success of a new transcatheter aortic valve replacement device: A surgeon's adventure
}

\author{
Amedeo Anselmi, MD, PhD, and Jean-Philippe Verhoye, MD, $\mathrm{PhD}$ \\ From the Division of Thoracic and Cardiovascular Surgery, Pontchaillou University Hospital, Rennes, France. \\ Disclosures: Authors have nothing to disclose with regard to commercial support. \\ Received for publication Sept 22, 2017; accepted for publication Sept 22, 2017; available ahead of print Nov 7 , \\ 2017. \\ Address for reprints: Amedeo Anselmi, MD, PhD, Division of Thoracic and Cardiovascular Surgery, Pontchaillou \\ University Hospital, 2, rue Henri Le Guilloux, Rennes 35 033, France (E-mail: amedeo.anselmi@alice.it). \\ J Thorac Cardiovasc Surg 2018;155:598-9 \\ $0022-5223 / \$ 36.00$ \\ Copyright (C) 2017 by The American Association for Thoracic Surgery \\ https://doi.org/10.1016/j.jtcvs.2017.09.113
}

Implanted for the first time in a human patient in 2014, the J-Valve (JC Medical, Burlingame, Calif) transcatheter aortic valve replacement (TAVR) concept has scored significant advancements. In perspective, it can be an attractive option for TAVR in aortic regurgitation, with a good deal of potential for success. The current article ${ }^{1}$ represents the first report of early and follow-up outcomes in a significant number of patients treated with this device. Unquestionably, this experience remains limited in terms of number of implants and amount of patient-years of follow-up. Yet, some prominent aspects deserve to be underlined.

First, this technology has been developed through the contribution of cardiac surgeons, who also currently are its only users, testifying once again on the vitality and the propensity toward evolution of our discipline. Second, the separation of locators and valve - and their consequent deployment in different times-represents a typical feature and, potentially, an effective means to improve immediate results (perivalvular leakage, anatomically coherent positioning), especially in aortic regurgitation cases. Nonetheless, the rate of perivalvular leakage reported in this article remains important and, not surprisingly, greater in aortic stenosis cases. It appears that the effectiveness of this TAVR concept relies on optimal landing of the locators at the nadir of each native aortic cusp: Irregular calcifications in aortic stenosis reasonably represent an anatomic obstacle in several cases. Missed positioning of the locators, as acknowledged by the authors, is at the root of many instances of paravalvular leakage in both regurgitation and stenosis cases. To such respect, bicuspid valve anatomy can be considered as a foe for the success of this TAVR system, including in the aortic regurgitation cases for which it was originally intended. Because it is a crucial issue, the landing of the locators at the cusps' nadirs cannot be based on tactile feedback alone but requires effective image-based guidance. Real-time 3-dimensional (3D) transesophageal echocardiography (TEE) may prove to be the best-suited modality and even essential to such scope. a TAVR device. essential.

\section{References} 2018; 155:588-97.

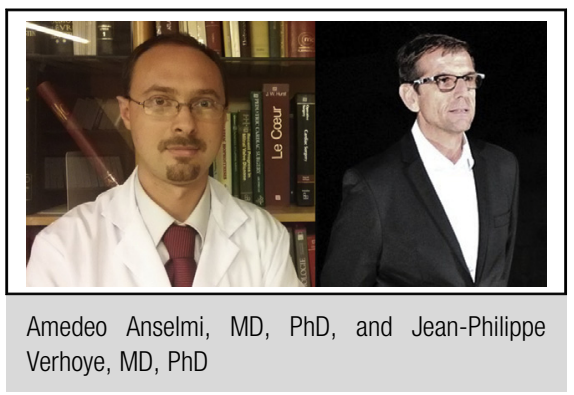

Central Message

A novel TAVR device needs to take the next steps toward success: enhanced image guidance and demonstration of reproducibility.

See Article page 588

3D TEE has demonstrated far greater accuracy and fineness than fluoroscopy and angiography in the intraprocedural evaluation of the mechanism of aortic regurgitation after deployment of a TAVR device. Real-time 3D TEE provides information about the degree of elliptical expansion of the valve device and the anatomic relationship between the prosthetic and native commissures (antianatomic expansion). ${ }^{2}$ All of this information can be discriminating in intraprocedural decision-making. Also, TEE has been proposed as the primary imaging modality to guide the deployment of

In transcatheter valve therapy, success is achieved through effectiveness and reproducibility. The pathway toward success of this promising TAVR concept will probably demand not only technologic advancements (miniaturization of the $27 \mathrm{~F}$ introducer, availability for retrograde delivery routes) but also full exploitation of intraprocedural 3D TEE capabilities, and, most important, proof of reproducibility in larger international trials. Cooperation with allied heart team professionals (anesthesiologist, sonographer cardiologist) will be

In the end, this pathway further stimulates us to take advantage of the surgical knowledge of cardiac anatomy, physiology, and pathology to continue being the cutting edge in the innovation for valve therapy.

1. Zhu L, Guo Y, Wang W, Liu H, Yang Y, Wei L, et al. Transapical transcatheter aortic valve replacement with a novel transcatheter aortic valve replacement system in high-risk patients with severe aortic valve diseases. J Thorac Cardiovasc Surg. 
2. Shibayama K, Mihara H, Jilaihawi H, Berdejo J, Harada K, Itabashi Y, et al. 3D assessment of features associated with transvalvular aortic regurgitation after TAVR: a real-time 3D TEE study. JACC Cardiovasc Imaging. 2016;9: 114-23.
3. Verhoye JP, Lapeze J, Anselmi A, Donal E. Association of transaortic approach and transoesophageal echocardiography as the primary imaging technique for improved results in transcatheter valve implantation. Interact Cardiovasc Thorac Surg. 2012;15:756-8. 